\title{
Le point sur la ventilation mécanique invasive - Principaux modes ventilatoires en ventilation mécanique invasive chez l'adulte
}

\author{
Latest developments for invasive mechanical ventilation - Main ventilatory \\ modes using invasive mechanical ventilation for adults
}

\author{
A. Roch · E. Mercier-IDE \\ (C) SRLF et Springer-Verlag France 2011
}

\section{Introduction}

Il existe de très nombreux modes ventilatoires. Parmi eux, certains sont de moins en moins utilisés, notamment ceux qui associent ventilation contrôlée (VC) et ventilation spontanée (modes dits « intermittents » comme la VACI ou la SIMV) [1], alors que de nouveaux modes sont en cours d'évaluation. Ces nouveaux modes, spontanés le plus souvent, sont beaucoup plus élaborés et pourraient trouver leur intérêt dans l'accélération du sevrage ventilatoire, en permettant automatiquement l'adaptation du niveau de ventilation aux besoins du patient, en analysant par exemple le signal EMG diaphragmatique (NAVA) [2] ou les paramètres respiratoires (fréquence, volume, $\mathrm{CO}_{2}$ expiré comme avec l'utilisation du SmartCare [3]). Cependant, les enquêtes de pratique, même récentes, montrent que les modes ventilatoires les plus couramment utilisés restent la ventilation assistée contrôlée (VAC) [en volume ou en pression] et la ventilation assistée avec aide inspiratoire. Dans le premier, la quasitotalité du travail ventilatoire du patient est prise en charge par le respirateur, car le patient est lourdement sédaté ou présente une défaillance neurologique ou musculaire. Dans le second, des cycles spontanés avec une aide en pression sont délivrés à un patient en cours de sevrage respiratoire. Ce chapitre décrira ainsi ces modes ventilatoires « traditionnels », dans leurs principes, leurs réglages et leur surveillance.

\footnotetext{
A. Roch $\cdot$ E. Mercier-IDE

Service de réanimation médicale,

détresses respiratoires et infections sévères,

hôpital Nord,

chemin des Bourrely,

F-13015 Marseille,

France
}

\section{Principes des modes ventilatoires et réglages}

\section{VC et VAC en volume}

Un volume déterminé (volume courant, Vt) est administré au patient à une fréquence respiratoire (FR) réglée (cycles contrôlés), et lorsque le ventilateur détecte un effort inspiratoire (cycles assistés, cf. « Déclenchement de l'inspiration - trigger inspiratoire »). Le volume pulmonaire dépend du sexe et de la taille. C'est pour cela que le Vt est réglé en fonction du poids idéal du patient $(8-10 \mathrm{ml} / \mathrm{kg})$ et non pas du poids mesuré. Le Vt est réduit en cas de lésions pulmonaires (ALI/SDRA) à 6-8 ml/kg. En utilisant des Vt trop élevés, on expose le patient à une surdistension alvéolaire responsable de baro- et volotraumatisme. Les risques d'un Vt trop bas sont le dérecrutement alvéolaire et donc l'hypoxémie et, par ailleurs, la désadaptation du patient du respirateur imposant l'augmentation de la sédation.

La FR est réglée afin d'obtenir une ventilation par minute $(\mathrm{Vt} \times \mathrm{FR})$ permettant d'obtenir un $\mathrm{pH}$ plus qu'une $\mathrm{PaCO}_{2}$ proche de la normale dans le sang, en pratique entre 10 et $35 /$ minute.

Le Vt est administré à un débit préréglé, c'est-à-dire à une certaine vitesse. Si le Vt est de 0,5 1 et le débit de 30 1/min, alors le $\mathrm{Vt}$ est administré en une seconde. En règle générale, le débit est réglé entre 40 et $80 \mathrm{l} / \mathrm{min}$.

Lors de l'administration du Vt, la pression dans les voies aériennes augmente progressivement, jusqu'à atteindre une pression de crête (ou pression de pic ou pression maximale) (Fig. 1). Cette pression de crête dépend du Vt administré (augmente avec le Vt) mais dépend aussi des voies aériennes du patient, qui opposent une certaine résistance (la pression de crête augmente si les voies aériennes sont rétrécies ou obstruées). On doit bien comprendre que la pression n'est donc pas réglée dans ce mode. La plupart des respirateurs permettent cependant de limiter la pression. Dans ce cas, si 


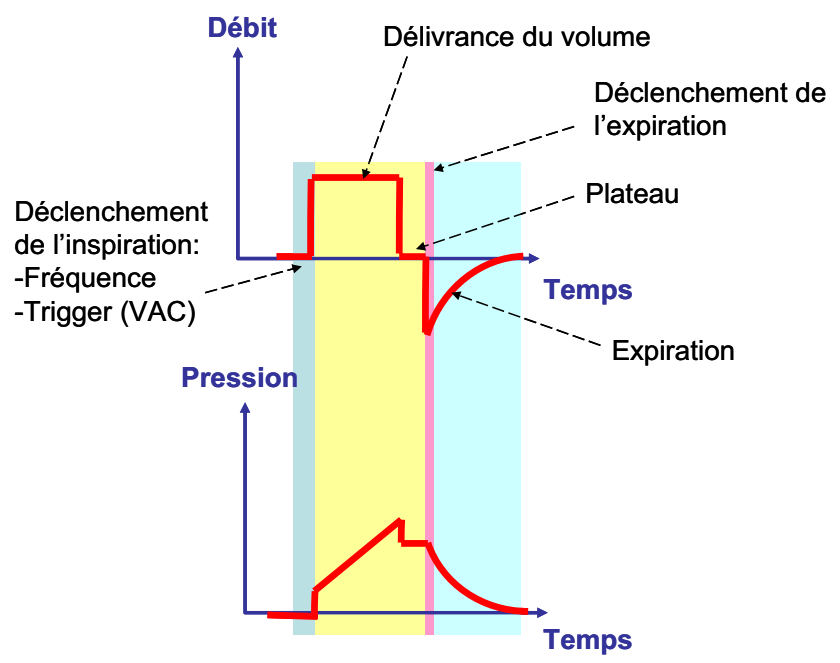

Fig. 1 Les phases du cycle ventilatoire en ventilation contrôlée en volume, sur la courbe de débit en fonction du temps et de pression en fonction du temps

la pression limite réglée est atteinte avant que le Vt réglé ait été complètement délivré, il y aura une diminution $\mathrm{du} \mathrm{Vt}$ administré. Ce réglage de pression limite présente peu d'intérêt en pratique pour limiter les risques d'hyperpression dans les alvéoles et donc de pneumothorax par exemple. En effet, la pression de crête reflète la pression qui règne dans les grosses bronches, et non pas dans les alvéoles, là où elle est potentiellement plus délétère si elle est élevée.

Le plus souvent, on fait suivre la phase d'administration du Vt par une phase dite de plateau. Pendant ce temps, aucun volume n'est administré, mais il n'y a pas d'expiration possible (valves du respirateur bloquées, état stable). Pendant ce temps de plateau, la pression qui règne dans les grosses bronches s'équilibre grossièrement avec celle qui règne dans les alvéoles. Elle n'a pas d'intérêt démontré sur la fonction respiratoire. Par contre, à la fin de ce temps, on peut mesurer la pression de plateau (Pplat), qui reflète grossièrement la pression qui règne dans les alvéoles pulmonaires à la fin de l'inspiration. C'est cette pression qui peut être délétère pour les alvéoles si elle est trop élevée, et l'utilisation d'une Pplat inférieure à $28-30 \mathrm{cmH}_{2} \mathrm{O}$ est recommandée, d'autant plus que les poumons sont lésés. La plupart des respirateurs mesurent et affichent cette Pplat si un temps de plateau a été réglé. La durée du temps de plateau est généralement de 0,2 à 0,5 seconde. Ainsi, le temps inspiratoire correspond à l'addition du temps de délivrance du Vt et du temps de plateau.

À la phase inspiratoire fait suite la phase expiratoire, qui est passive. En VC en volume, l'expiration est déclenchée lorsque le Vt a été administré ou lorsque la pression limite a été atteinte. Il est d'autant plus important de respecter un temps expiratoire suffisant que les poumons du patient mettront beaucoup de temps à expirer le volume insufflé, notamment chez les patients avec BPCO, dont les

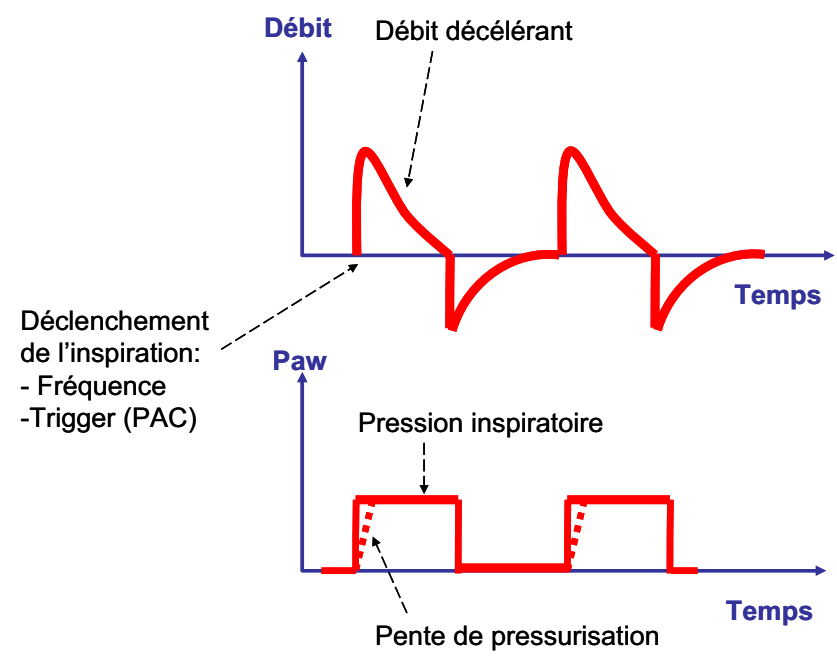

Fig. 2 Les phases du cycle ventilatoire en ventilation contrôlée en pression, sur la courbe de débit en fonction du temps et de pression en fonction du temps

bronches sont enflammées et comprimées par des poumons distendus.

\section{$\mathrm{VC}$ et VAC en pression}

En ventilation à pression contrôlée (PC) ou assistée à $\mathrm{PC}$, une pression constante est appliquée aux voies aériennes pendant la phase inspiratoire (Fig. 2), à une fréquence réglée (cycles contrôlés) ou lorsque le ventilateur détecte un effort inspiratoire (cycles assistés, cf. « Déclenchement de l'inspiration - trigger inspiratoire $»)$. On règle un temps inspiratoire, correspondant au temps pendant lequel le ventilateur pressurise les voies aériennes. Entre les inspirations, la pression des voies aériennes correspond à la pression expiratoire positive (PEP).

Dans ce mode, le volume n'est pas prédéterminé. Il va varier en fonction des paramètres réglés (pression inspiratoire, PEP, temps inspiratoires et expiratoires, pente de montée en pression), des caractéristiques mécaniques du système respiratoire du patient (résistance inspiratoire, compliance, PEP intrinsèque) et de ses efforts inspiratoires pour les cycles assistés (intensité, durée). Notamment, toute diminution de la compliance respiratoire (atélectasie, pneumothorax) ou toute augmentation des résistances inspiratoires (encombrement, bronchospasme, sonde bouchée) se traduit par une diminution du volume inspiré en mode à PC.

Le débit inspiratoire est maximal en début d'inspiration puis décroît exponentiellement (débit décélérant). Le débit inspiratoire devient nul d'autant plus vite que les résistances et compliance du système respiratoire sont élevés.

La pente de montée en pression correspond à la vitesse à laquelle le respirateur pressurise les voies aériennes. Plus la pente est rapide, plus importante est l'assistance délivrée. 
À Vt égal, la pression de crête est plus basse en PC qu'en mode à volume contrôlée, en raison du débit décélérant qui réduit la pression résistive au cours de l'inspiration. Cependant, la Pplat, reflet de la pression alvéolaire maximale, n'est en rien modifiée [4]. La génération de cycles spontanés chez les patients ayant une activité inspiratoire soutenue peut même générer des $\mathrm{Vt}$ très élevés, source de lésions alvéolaires potentielles.

\section{Ventilation spontanée avec aide inspiratoire (VSAI)}

C'est un mode en pression. Lorsque le ventilateur décèle l'effort inspiratoire (cf. " Déclenchement de l'inspiration - trigger inspiratoire »), le ventilateur pressurise les voies aériennes au niveau réglé, cette pression restant constante au cours de l'inspiration entière. Le niveau d'assistance est déterminé par le niveau d'aide inspiratoire (différence entre pression inspiratoire et PEP). Ainsi, le niveau d'aide déterminera le Vt délivré au patient. En augmentant l'aide, on observe généralement une diminution de la FR associée à une augmentation du Vt, permettant ainsi d'optimiser le niveau d'aide au confort du patient. La pente de montée en pression correspond à la vitesse à laquelle le respirateur pressurise les voies aériennes. Plus la pente est rapide, plus importante est l'assistance délivrée. La durée du temps inspiratoire dépendra de l'évolution du débit inspiratoire au cours du cycle (cf. " Déclenchement de l'expiration cyclage - trigger expiratoire »).

\section{BIPAP (biphasic positive airway pressure)- APRV (airway pressure release ventilation)}

Ces deux appellations correspondent globalement au même mode ventilatoire, au cours duquel le ventilateur fait varier à une fréquence réglée la pression des voies aériennes entre un niveau bas (assimilable à la PEP) et un niveau haut (pression inspiratoire), tout en laissant libre le patient d'effectuer des cycles spontanés à tout moment. Si le patient n'a pas de cycles spontanés, la BIPAP-APRV est équivalente à la ventilation en PC. Si le patient a une activité spontanée superposée, celle-ci peut faire l'objet d'une aide inspiratoire. Ce mode permet notamment de progresser vers le sevrage en nécessitant des moindres niveaux de sédation qu'en VAC.

\section{Autres réglages}

\section{Déclenchement de l'inspiration - trigger inspiratoire}

Ce déclenchement (ou trigger) se fait de manière contrôlée en fonction de la FR en mode de VC ou de PC. Dans ces modes, aucun cycle supplémentaire ne peut être délivré. En mode de VAC ou de pression assistée contrôlée (PAC ou VPC), des cycles supplémentaires peuvent être déclenchés par le patient. Dans ce cas, le respirateur détectera soit une dépression dans les voies aériennes induite par des mouvements des muscles inspiratoires (trigger en pression), soit un débit entrant dans les voies aériennes (trigger en débit), là aussi induit par des mouvements des muscles inspiratoires [5]. Lors de la détection de ce trigger, le ventilateur administre un Vt équivalent à celui délivré lors des cycles contrôlés (VAC) ou pressurise les voies aériennes pendant un temps inspiratoire et à un niveau préréglé (PAC-VPC). La plupart des respirateurs permettent le réglage du niveau de pression ou du débit de trigger inspiratoire, de façon à plus ou moins faciliter l'administration de cycles supplémentaires. L'administration de ces cycles supplémentaires facilite l'adaptation du patient au respirateur et l'adéquation entre demande ventilatoire et apport par la machine, constituant une première étape vers le sevrage. En pratique, le trigger inspiratoire doit être réglé au niveau le plus bas compatible avec l'absence d'autodéclenchements, qui sont des fluctuations du niveau de pression expiratoire (entre les inspirations) qui sont interprétées à tort par la machine comme des efforts inspiratoires du patient (en raison de fuites ou d'une instabilité du contrôle du niveau de PEP par la machine). En VSAI, la ventilation n'étant constituée que de cycles assistés, le réglage efficace du trigger inspiratoire est encore plus crucial.

\section{Déclenchement de l'expiration - cyclage - trigger expiratoire}

En VC ou en VAC, le déclenchement de l'expiration est réglé, car le temps inspiratoire est fixe. En $\mathrm{VC}$ en volume (VC-VAC), l'expiration se déclenche lorsque le Vt a été administré. En ventilation en PC (PC-PAC-VPC), l'expiration se déclenche à la fin du temps inspiratoire préréglé.

En VSAI, l'expiration se déclenche lorsque le débit inspiratoire, qui est décélérant au cours de l'inspiration, descend en dessous d'un certain pourcentage du débit maximal atteint. Sur la plupart des respirateurs, le déclenchement est réglé à $25 \%$ du débit inspiratoire maximal. Sur d'autres respirateurs, cette consigne est réglable, de 5 à $50 \%$ en général (Fig. 3). Plus le pourcentage est bas, plus la fin de l'inspiration sera tardive et donc plus le temps inspiratoire sera long et le Vt important.

\section{Rapport I/E}

Le rapport I/E est le rapport du temps inspiratoire au temps expiratoire. Physiologiquement, il est de l'ordre de $1 / 2$, c'est-à-dire que l'expiration est deux fois plus longue que l'inspiration (temps inspiratoire $=33 \%$ de la durée du cycle, temps expiratoire $=66 \%$ ).

À Vt constant et débit constant, une augmentation de la FR réduira le temps dévolu chaque minute à l'expiration, donc augmentera le rapport I/E. D'un autre côté, une augmentation du débit inspiratoire (Fig. 4) ou une réduction du 


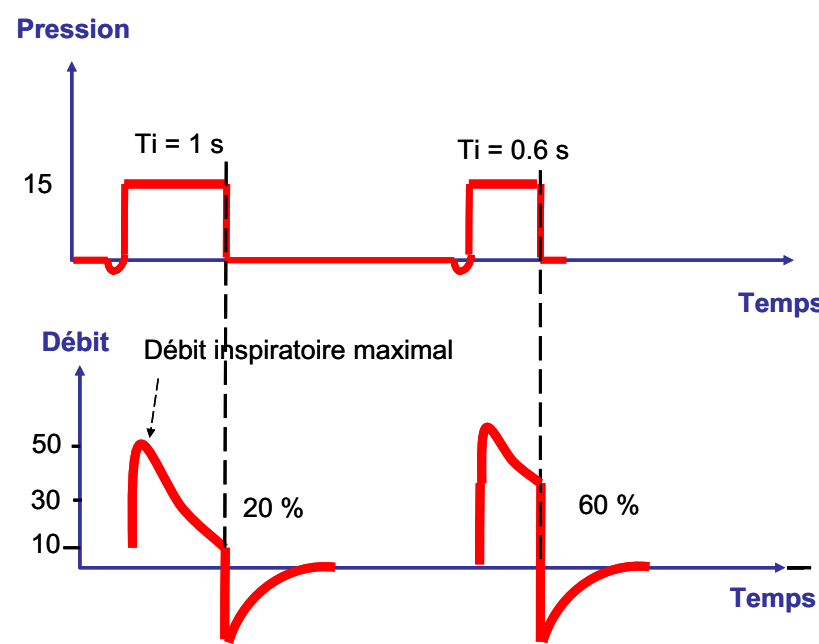

Fig. 3 En ventilation spontanée avec aide inspiratoire (VSAI), effet du réglage du trigger expiratoire sur le temps inspiratoire (Ti). En passant de 20 à $60 \%$ du débit expiratoire maximal, on observe une diminution du temps inspiratoire

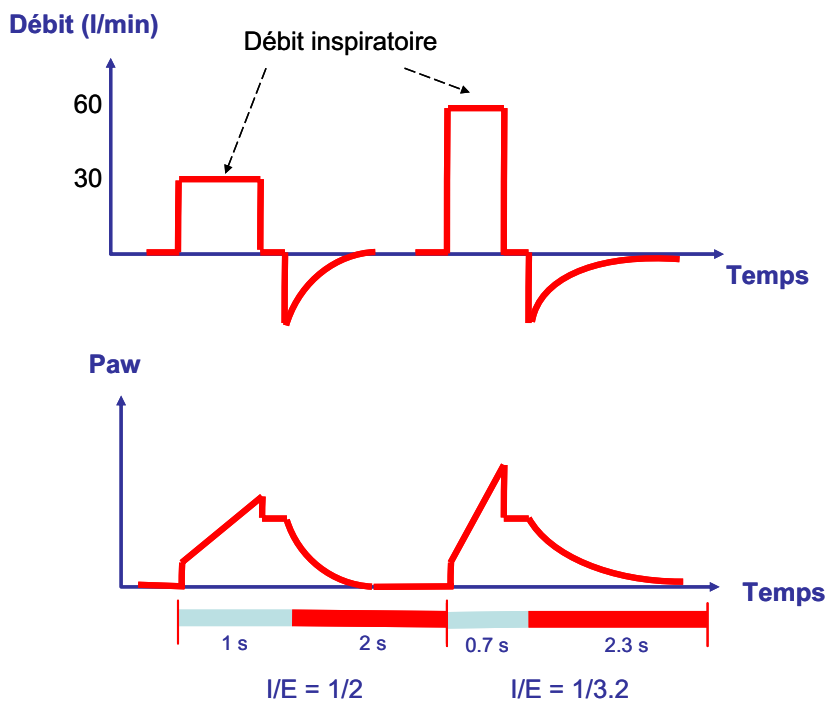

Fig. 4 En VAC, effet d'une augmentation du débit inspiratoire sur le rapport $\mathrm{I} / \mathrm{E}$

Vt raccourcira l'inspiration et diminuera le rapport I/E. Ainsi, chez les patients avec BPCO, chez qui, on souhaite allonger le temps expiratoire, on augmentera généralement le débit inspiratoire en diminuant la FR, de façon à atteindre un rapport I/E de l'ordre de 1/5.

\section{PEP}

Le réglage de la PEP est commun à tous les modes ventilatoires. En l'absence de PEP, la pression dans les voies aériennes revient à zéro lors de l'expiration. Schématiquement, le but de la PEP est d'empêcher la fermeture en expiration d'alvéoles qui ont tendance à cette fermeture, car les poumons sont lésés (inflammation, œdème, altération du surfactant qui, en conditions physiologiques, maintient les alvéoles ouvertes à l'expiration, favorisant ainsi l'oxygénation et empêchant des phénomènes délétères de fermeture et réouverture cyclique de ces alvéoles).

Sur la courbe de pression, l'augmentation de la PEP se traduit logiquement par une augmentation de la pression de crête lors de la phase inspiratoire. Même s'il n'existe pas de consensus sur le niveau optimal de PEP à utiliser chez les patients aux poumons lésés (ALI/SDRA), retenons globalement que la PEP est augmentée parallèlement à la profondeur de l'hypoxémie.

\section{Surveillance}

\section{En VC ou en VAC}

La détection d'événements mécaniques repose sur la surveillance des pressions de crête et de plateau. Ainsi, une augmentation de la pression de crête peut être le fait d'une augmentation des résistances inspiratoires (obstruction de sonde, bronchospasme, encombrement) si la Pplat est inchangée ou d'une diminution de la compliance du système respiratoire si la Pplat est augmentée (pneumothorax par exemple). À noter que le réglage de l'alarme de pression de crête, qu'on réglera à 10 à $20 \%$ au-dessus de la valeur notée en début de vacation, est différent du niveau de pression limite, qui correspond au niveau de pression auquel le ventilateur va déclencher l'expiration s'il est atteint. Ce niveau de pression limite n'a que peu d'intérêt en pratique, car une pression de crête élevée n'est pas en soi délétère, et qu'une pression limite expose au risque de délivrance partielle du Vt.

Les alarmes de Vt bas, de PEP basse, de pression inspiratoire basse et de volume minute bas ont pour but de détecter une déventilation du patient ou une fuite sur le circuit. Ces alarmes peuvent se déclencher simultanément ou de manière décalée en fonction du premier critère atteint.

L'alarme de fréquence haute a pour but de déceler, en VAC, une augmentation brutale de la demande ventilatoire du patient, en rapport avec un problème mécanique ou extrarespiratoire (réveil, douleur...).

\section{En PC ou en PAC}

Le Vt est le paramètre essentiel de surveillance de la ventilation en pression, car il n'est pas réglé ni garanti dans ce mode. Le réglage du Vt bas permet de déceler une augmentation de résistance (sonde obstruée, encombrement, bronchospasme) ou de compliance (atélectasie, pneumothroax), 
car dans ce cas, le Vt va diminuer. La diminution du volume minute n'est pas systématique dans ce cas, car la baisse du Vt peut être compensée par une augmentation de la FR. L'alarme de Vt haut permettra d'éviter la délivrance de volumes potentiellement délétères. Cette alarme est réglée à 10 à $20 \%$ au-dessus du Vt désiré ( $8 \mathrm{ml} / \mathrm{kg}$ de poids prédit).

\section{En VSAI}

Les alarmes sont identiques à celles de la ventilation assistée en PC. On y ajoute une surveillance de la FR basse, puisque aucun cycle n'est garanti dans ce mode (sauf lors du passage en ventilation d'apnée). Une FR basse peut révéler une déventilation (alarmes de Pi basse et de Vt bas associées), une obstruction complète de la sonde ou de la trachée (alarmes de Vt bas associées), une aide trop importante (alarme de Vt haut associée possible) ou une hypoventilation d'origine neurologique.
Conflit d'intérêt : les auteurs déclarent ne pas avoir de conflit d'intérêt.

\section{Références}

1. Esteban A, Ferguson ND, Meade MO, et al (2008) Evolution of mechanical ventilation in response to clinical research. Am J Respir Crit Care Med 177(2):170-7

2. Sinderby C, Navalesi P, Beck J, et al (1999) Neural control of mechanical ventilation in respiratory failure. Nat Med 5(12):1433-6

3. Dojat M, Harf A, Touchard D, et al (2000) Clinical evaluation of a computer-controlled pressure support mode. Am J Respir Crit Care Med 161(4 Pt 1):1161-6

4. Mercat A, Graïni L, Teboul JL, et al (1993) Cardiorespiratory effects of pressure-controlled ventilation with and without inverse ratio in the adult respiratory distress syndrome. Chest 104(3):871-5

5. Sassoon CS, Gruer SE (1995) Characteristics of the ventilator pressure - and flow-trigger variables. Intensive Care Med 21 (2):159-68 